Research Article

\title{
Research on Landslide Warning Model Establishment and Disaster Space-Time Evolution Analysis
}

\author{
Zhen Wang \\ School of Engineering, Huanghe Science and Technology College, Zhengzhou, Henan, China \\ Correspondence should be addressed to Zhen Wang; hhstu_wz@163.com
}

Received 7 April 2021; Revised 28 April 2021; Accepted 3 May 2021; Published 20 May 2021

Academic Editor: Faming Huang

Copyright (C) 2021 Zhen Wang. This is an open access article distributed under the Creative Commons Attribution License, which permits unrestricted use, distribution, and reproduction in any medium, provided the original work is properly cited.

\begin{abstract}
As important methods to avoid landslide disasters, velocity monitoring and early warning are significant research topics in slope engineering at the present stage. This paper combines the randomness of velocity data in evolution process of landslide disasters, using Markov chain theory with no aftereffect to describe the randomness process, and introduces it into landslide warning. The research collects velocity monitoring data before landslide occurrence and applies average standard deviation method which can reflect statistical characteristics of the classification data to carry out state division of the velocity data. Then, it proposes landslide warning criteria and establishes landslide warning model based on dynamic prediction of future velocity status by Markov chain theory. Meanwhile, it puts forward the evaluation standard of landslide warning model from the aspects of timeliness, antiinterference, and credibility. At the same time, it takes typical open-pit mine landslide disaster as the engineering background and gradually optimizes and evaluates the landslide warning model from the above three evaluation standards. The results show that the landslide warning model can realize the landslide early warning of multiple monitoring points; it has good effects in both time warning and regional warning. On the other hand, the landslide warning model has high accuracy in timeliness, anti-jamming, and credibility, and it can reveal space-time evolution law of landslide occurrence, so this research has important theoretical significance and engineering promotion value.
\end{abstract}

\section{Introduction}

The frequency of large geological disasters has increased significantly with the severe climate changing, the continuous increasing of global population, the gradual expansion of human living areas, the increase geological disturbances of engineering activity, and other influencing factors since the 20th century [1-3]. Among them, the quantity of landslide disasters accounted for more than $70 \%$ of the total amount of geological disasters, and it is becoming the highest occurrence frequent and the maximum economic losses of geological disasters $[4,5]$.

As important methods to avoid landslide disasters, velocity monitoring and early warning are significant research topics in slope engineering; its core is monitoring data collection and landslide warning analysis. In recent years, with the development of slope monitoring technology, monitoring data collection has been effectively solved, but how to establish a landslide warning model based on monitoring data is still an important problem in the field of slope engineering at this stage. The research on landslide warning began in the 1960s. Japanese scholar Saito proposed an empirical formula for landslide warning, creating a precedent for landslide warning research [6]. In the next few decades, scholars from various countries have successively proposed dozens of landslide warning models and have experienced development stages such as qualitative landslide warning, quantitative landslide warning, and comprehensive landslide warning [7-11]. Qualitative landslide warning established the slope catastrophic evolution model based on engineering experience, and then analyzed catastrophic stage and stability state of the slope [12]. Guo counted 119 landslide cases in Wenchuan earthquake and established a landslide warning model from the perspectives of horizontal peak, slope angle, ground acceleration, rock type, geological structure, and so on by qualitative analysis method [13]. 
Wang used qualitative analysis methods to establish a landslide warning model which comprehensively considered rainfall, river erosion, human activities, and other factors and evaluated the typical loess slope stability in Baoji City, Shaanxi Province [14]. Quantitative landslide warning is a method which numericalizes geological features and external environment in the process of slope catastrophe evolution and then analyzes and forecasts the slope stability by mathematical and physical methods [15]. Ciervo analyzed the change law of soil mechanics parameters in rainfallinduced landslide evolution by mathematical statistical method and proposed a landslide warning model based on the evolution of soil mechanics parameters. At the same time, he applied the model to the landslide warning on the Amalfi coast in southern Italy and achieved good prediction results [16]. Yan quantified the influence of external environment on slope stability through sensitivity analysis and established a quantitative landslide warning model which considered the influence of external environment [17]. Comprehensive landslide warning is a method which applied physical simulation, numerical simulation, and other means to establish a geological, mechanical, and deformation coupling model that comprehensively reflects internal mechanism and external performance in the process of landslide disasters [18]. Tiranti established a landslide warning model that comprehensively considers strength conditions and deformation constraints through physical simulation method, and applied the model into Piemonte regional landslide warning, which achieved good warning results [19]. Canli established a coupled hydromechanical model to describe the evolution process of landslide disasters in rainfalls and applied it into slope warning with rainfalls [20]. The majority of scholars have done lots of research on landslide warning model, but the research results are still not very ideal. First of all, landslide warning models are hysteresis and warning information is not timely enough; it is to say there is not enough time to prepare for the disaster before landslides occur [21]. Secondly, a large number of landslides occur outside the warning area, that is to say, the failure rate of landslide area is very high. Lastly, the error warning rate is high, that means the warning information is provided when there is no risk of landslides, and this error warning information will have a serious impact on the normal productions and lives of residents [22]. The reason is that, due to the complexity, randomness, and uncertainty of landslide disasters evolution, a warning model is only suitable for a certain type or a certain stage of landslide prediction, and various landslide warning models have certain limitations. In addition, landslide warning should include landslide time warning and landslide area warning, the previous research focused on landslide time warning, and the landslide area warning is insufficient, but these two aspects should be in mutual unity in fact [23].

In the evolution process of landslide disasters, the antisliding force changing with time can be regarded as a random process, and the velocity data obtained by slope monitoring can also be regarded as a random process correspondingly [24]. Using the theories and methods of random process analyzed data and forecast warning have solid theoretical foundation and realistic practical requirements. Markov chain theory, as a method to describe the law of transition between system states, describes a process that the probability regularity of future state has been determined if the current state of the system is known; that is to say, future state of the system has nothing to do with past state under the condition that current state is known [25]. This memoryless feature is consistent with the practice of predicting future stability of the slope based on current stability in landslide warning, and it reflects the feasibility of Markov chain theory in landslide warning [26]. Therefore, this paper made an intensive research of landslide warning method based on Markov chain theory and established a reasonable and accurate landslide warning model to provide scientific means and theoretical basis for landslide disasters warning.

\section{Landslide Warning Method}

Markov chain theory is a method that describes the law of transition between system states; applying the method can obtain the transfer probability between different states and thus can predict the future state change trend of the system.

2.1. Markov Chain Theory. Assume random process $X(n),\{X(n), n \in N\}$, where $N$ is the set of nature numbers, and all values of $X(n)$ make up the discrete state $I, I=$ $\left\{i_{0}, i_{1}, i_{2}, i_{3} \ldots\right\}$, if the conditional probability satisfies formula (1) for any arbitrary $n \in N$ and $i \in I$,

$$
P\left\{X(n+1)=i_{n+1} X(n)=i_{n}, X(n-1)=i_{n-1}, X(n-2)=i_{n-2} \ldots X(0)=i_{0}\right\}=P\left\{X(n+1)=i_{n+1} X(n)=i_{n}\right\}
$$

Then, we call $\{X(n), \quad n \in N\}$ as a Markov chain. The process of $X(t),\{X(t), t \in T\}$, is a random vector that satisfies Markov chain and contains time factors when the set of nature numbers in formula (1) is endued to time attribute. 


$$
\begin{aligned}
& P\left\{X(t)=i_{t}, X(t-1)=i_{t-1}, X(t-2)=i_{t-2} \ldots X(0)=i_{0}\right\} \\
&=P\left\{X(t)=i_{t} \mid X(t-1)=i_{t-1}, X(t-2)=i_{t-2}, X(t-3)=i_{t-3} \ldots X(0)=i_{0}\right\} \\
& \cdot P\left\{X(t-1)=i_{t-1}, X(t-2)=i_{t-2}, X(t-3)=i_{t-3} \ldots X(0)=i_{0}\right\} \\
&= P\left\{X(t)=i_{t} \mid X(t-1)=i_{t-1}\right\} \cdot P\left\{X(t-1)=i_{t-1}, X(t-2)=i_{t-2}, X(t-3)=i_{t-3} \ldots X(0)=i_{0}\right\} \\
&= \cdots \\
&= P\left\{X(t)=i_{t} \mid X(t-1)=i_{t-1}\right\} \cdot P\left\{X(t-1)=i_{t-1} \mid X(t-2)=i_{t-2}\right\} \\
& \cdot P\left\{X(t-2)=i_{t-2} \mid X(t-3)=i_{t-3}\right\} \ldots P\left\{X(1)=i_{1} \mid X(0)=i_{0}\right\} .
\end{aligned}
$$

In formula (2), $X(t)$ is the state of moment $t$, and finite moment $t$ forms a time set $T, T=\{0,1,2,3 \ldots\}$. It is obvious from formula (2) that the statistical properties of Markov chain are determined by the conditional probability $P\left\{X(t+1)=i_{t+1} \mid X(t)=i_{t}\right\}$. The conditional probability means the probability of system state is $i_{t+1}$ at moment $t+1$ under the condition that system state is $i_{t}$ at moment $t$. Therefore, the probability distribution of future states can be determined under the premise that initial state probability vector and transition probability matrix are known, so that reasonable predictions can be made of future states.

2.2. Markov Chain Prediction Method. For a data sequence composed of multiple data $\left\{x_{1}, x_{2}, x_{3} \cdots x_{n}\right\}$, the data is divided into $r$ states according to a specific standard, and $q_{i j}$ represents the times of state sequence transitions from state $i$ to state $j$ by $k-1$ steps ( $k$ is the number of statistical steps, $k=1,2,3 \cdots m)$. Obviously, $q_{i j}$ can form an $n \times n$ state transition frequency matrix $q^{(k)}$. Formula (3) can be used to calculate the state transfer probability matrix $p^{(k)}$ according to the state transition frequency matrix $q^{(k)}$.

$$
p_{i j}=q_{i j} \cdot\left(\sum_{i=1}^{i=r} q_{i j}\right)^{-1} \text {. }
$$

For an arbitrary state $l, l \in\{1,2,3 \cdots r\}, p_{l}$ is 1 when the state of $x_{n-k+1}$ is $l$. On the other side, $p_{l}$ is 0 when the state of $x_{n-k+1}$ is not $l$. Thus, the initial state probability vector $A_{k}^{T}$,
$A_{k}^{T}(n)=\left(p_{1}, p_{2}, p_{3} \ldots p_{l} \ldots p_{r}\right)$, can be determined when the step length is $k$. So the state distribution matrix $B_{k}^{(n+1)}$ of $x_{n+1}$ can be expressed as formula (4) in the condition of the step length being $k$.

$$
B_{k}^{(n+1)}=A_{k}^{T}(n) \cdot p^{(k)}
$$

For the different values of $k, B_{k}^{(n+1)}$ consists of matrix $B, B=\left(B_{1}^{(n+1)}, B_{2}^{(n+1)}, B_{3}^{(n+1)} \cdots B_{m}^{(n+1)}\right)^{T}$. The calculation formula of self-correlation coefficient $r_{k}$ can be expressed as formula (5) when the step length is $k$.

$$
\begin{aligned}
r_{k}= & {\left[\sum_{t=1}^{t=n-k}\left(x_{t}-x\right)\left(x_{t+k}-x\right)\right] } \\
& \cdot\left[\sum_{t=1}^{t=n-k}\left(x_{t}-x\right)^{2} \cdot \sum_{t=1}^{t=n-k}\left(x_{t+k}-x\right)^{2}\right]^{-(1 / 2)} .
\end{aligned}
$$

In formula (5), $x$ is average value of the data $\left\{x_{1}, x_{2}, x_{3} \ldots x_{n}\right\}$. Accordingly, the weight vector $w_{k}$ with step length $m$ can be calculated by

$$
w_{k}=\left|r_{k}\right| \cdot\left(\sum_{k=1}^{k=m}\left|r_{k}\right|\right)^{-1} .
$$

Different step weights form an $m \times m$ weight matrix $w$, $w=\left(w_{1}, w_{2}, w_{3} \ldots w_{k} \ldots w_{m}\right)$. The probability distribution vector $C$ of next day prediction state through weighted Markov chain can be expressed as

$$
C^{(n+1)}=\left(c_{1}, c_{2} \ldots c_{r}\right)=\left(w_{1}, w_{2} \ldots w_{k} \ldots w_{m}\right) \cdot\left(B_{1}^{(n+1)}, B_{2}^{(n+1)} \ldots B_{m}^{(n+1)}\right)^{T}
$$

In the probability distribution vector $C$, the state corresponding to the column where the largest element is located is the most probable state for $x_{n+1}$ predicted by the weighted Markov chain.

2.3. Markov Chain Landslide Warning Model. According to Markov chain prediction method, the state sequence contains time information of velocity data change if velocity data obtained by slope monitoring is divided into several states through certain criteria. Then, we can establish statistical laws of the slope stability state changes over time. Combined with velocity monitoring data and future state prediction characteristics, the model can determine whether to provide warning information. The establishment of landslide warning model can be expressed as follows.

2.3.1. Status Classification of Velocity Data. Landslide warning model should make a clear judgment on safety states of the slope stability. At the same time, states division should follow the principle of concise and clear. Therefore, velocity data was divided into two states of safe and dangerous. For the monitoring velocity data, the average value $x$ and standard deviation $s$ were calculated, and the boundary point $D$ was determined according to $D=x+b \cdot s$ ( $b$ is a 
variable parameter and it represents standard deviation multiple). It was defined as safe and represented by " 1 " when the velocity data was in the interval $\left[x_{\text {min }}, D\right)$. Conversely, it was defined as dangerous and represented by " 2 " when the velocity data was in the interval $\left[D, x_{\max }\right]$.

2.3.2. Dynamic Prediction of Future Velocity. In order to effectively reduce the error warning rate of landslide warning model, the comprehensive prediction results are defined dangerous only in the condition that future states prediction results for the step length 1, 2, 3, 4, 5 of Markov chain prediction method are all dangerous. At the same time, the importance of current data is highlighted and the influence of historical data is gradually diminishing with the continuous update of monitoring data. As a result, the warning model should eliminate early historical data while incorporating new data opportunely and predicts the future velocity state constantly through Markov chain prediction method.

2.3.3. Establishment of Landslide Warning Criteria. The warning model should also make clear whether to provide warning information while predicting the future velocity state. In the phase of constant velocity deformation, the dangerous velocity is rare and the prediction results will inevitably show a large number of safe states. Even if the dangerous state occurs, it will gradually revise and restore to safe state in dynamic data updating. On the other side, in the phase of accelerated velocity deformation before landslide, dangerous velocity appears in large numbers and the prediction results will continue to show dangerous values. The prediction will usually not revise and restore to safe state in the dynamic data updating due to the irreversible of landslides. Therefore, the landslide warning criteria of Markov chain landslide warning model can be described as follows:

The actual velocity state of yesterday was dangerous

The actual velocity state of today is dangerous Landslide warning $\longrightarrow$ Landslide warning.

The prediction velocity state of next day is safe

2.4. Evaluation Index of Landslide Warning Model. In order to provide necessary emergency preparation time for people evacuation and property transfer, the basic requirement of landslide warning model is to give frequent warning information before the occurrence of landslides. At the same time, to ensure normal production and life of residents, the landslide warning model should not give warning information when there is no risk of landslide. Aiming at the above goals, this paper evaluates the landslide warning model from three aspects: timeliness, anti-interference, and credibility.

Apply warning intensity $I$ to evaluate the index of timeliness. It is defined that, in $R$ times of warning judgments within landslide emergency preparation time, warning model gives $R *$ times of warning information; then, the warning intensity $I=R * / R$. Apply error warning rate $W$ to evaluate the index of anti-interference. It is defined that, in $M$ times of warning judgments before landslide emergency preparation time, warning model gives $M *$ times of warning information; then, the error warning rate $W=M^{*} / M$. Apply prediction consistency rate $Y$ to evaluate the index of credibility. It is defined that, in $Q$ times of warning judgments, $Q *$ times are consistent with the actual state among them; then, the prediction consistency rate $Y=Q * / Q$. Among the above three indexes, timeliness is the most significant evaluation index, anti-interference is an optimization index under the premise of satisfying timeliness, and credibility is an evaluation of model accuracy accumulation under the premise of satisfying timeliness and anti-interference. There is no need to pursue excessive credibility in engineering practice.

\section{Engineering Case}

3.1. Slope Engineering Overview. A large-scale landslide occurred in an open-pit mine on April 17. For the landslide body, the length is more than $700 \mathrm{~m}$, the width is nearly $400 \mathrm{~m}$, the height is nearly $200 \mathrm{~m}$, and the volume is more than 3 million $\mathrm{m}^{3}$. There are 28 velocity monitoring points at 438, 462, 512, and 548 levels near the landslide area. Among them, 21 monitoring points are located in the landslide area and 7 monitoring points are located outside the landslide area [27]. The prospect of landslide area and the layout of monitoring points are shown in Figure 1.

3.2. Landslide Warning Analysis. This paper selected 5 January to 17 April as monitoring period. At the same time, due to the huge amount of data, we selected WY3250-461 monitoring point and used sample size of 20 and standard deviation multiple of 0.4 to illustrate the prediction process. The velocity monitoring data of WY3250-461 monitoring point in sample size of 20 is shown in Table 1 .

The minimum velocity is $0.44 \mathrm{~mm} \mathrm{~d}^{-1}$ and the maximum velocity is $3.02 \mathrm{~mm} \mathrm{~d}^{-1}$ for the 20 velocity data in Table 1 . At the same time, the average value $x$ is 1.745 and the standard deviation $s$ is 0.675 . It can determine that the boundary point $D$ is 2.015 in the condition of standard deviation multiple is 0.4 based on the formula $D=x+b \cdot s$. Therefore, it is defined safe and represented by " 1 " when the velocity data is in the interval $[0.44,2.015)$, and it is defined dangerous and represented by " 2 " when the velocity data is in the interval $[2.015,3.02]$. The state transition probability matrix with step length of $1,2,3,4,5$ can be calculated according to 

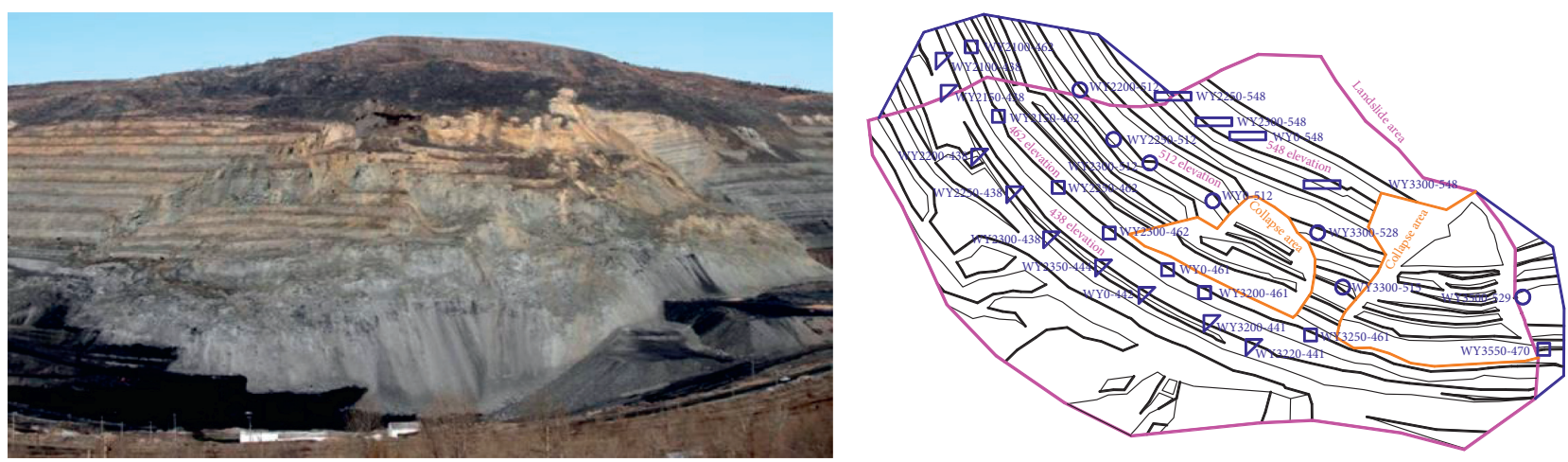

Boundary lines of landslide area Boundary lines of collapse area Top lines of step slope

Bottom lines of step slope

Monitoring points at 438 elevation

Monitoring points at 462 elevation

Monitoring points at 512 elevation

Monitoring points at 548 elevation

(a)

(b)

Figure 1: Engineering overview of landslide area. (a) Prospect of landslide area. (b) Layout of monitoring points.

TABLE 1: WY3250-461 monitoring point velocity data.

\begin{tabular}{|c|c|c|c|c|c|c|c|c|c|c|}
\hline Date & 5 Jan & $6 \mathrm{Jan}$ & 7 Jan & 8 Jan & 9 Jan & 10 Jan & 11 Jan & 12 Jan & 13 Jan & 14 Jan \\
\hline Velocity $/ \mathrm{mm} \cdot \mathrm{d}^{-1}$ & 1.53 & 1.37 & 2.24 & 2.58 & 1.54 & 2.43 & 0.44 & 1.59 & 1.78 & 1.93 \\
\hline Date & 15 Jan & 16 Jan & 17 Jan & 18 Jan & 19 Jan & 20 Jan & 21 Jan & 22 Jan & 23 Jan & 24 Jan \\
\hline Velocity $/ \mathrm{mm} \cdot \mathrm{d}^{-1}$ & 1.68 & 1.34 & 1.98 & 1.39 & 0.68 & 3.00 & 0.98 & 1.66 & 1.74 & 3.02 \\
\hline
\end{tabular}

formula (3). For this example, the 20th velocity data state is 2 , and the initial state vector $A_{1}^{T}$ is $(0,1)$. Similarly, the 19 th, 18 th, 17 th, and 16 th velocity data state are, respectively, 1,1 , 1,2 , and the initial state vectors are, respectively, $A_{2}^{T}=(1,0), A_{3}^{T}=(1,0), A_{4}^{T}=(1,0), A_{5}^{T}=(0,1)$. The selfcorrelation coefficient and weight vector with step length of $1,2,3,4,5$ can be calculated through formulas (5) and (6). On this basis, the probability distribution vector of future velocity state with each step length can be obtained. It can be known that the probability of predicting future velocity state as safe is 0.833 with step length of 1 , the probability of predicting future velocity state as safe is 0.758 with step length of 2 , the probability of predicting future velocity state as safe is 0.672 with step length of 3 , the probability of predicting future velocity state as safe is 0.695 with step length of 4 , and the probability of predicting future velocity state as safe is 0.743 with step length of 5 . Therefore, Markov chain prediction method predicts that the future velocity state is safe.

Dynamically update data and implement Markov chain prediction process after acquiring new data so that we can obtain the state prediction sequence. According to actual velocity state information and predicted velocity state information, a warning judgment can be made based on landslide warning criteria. For WY3250-461 monitoring point, landslide warning model gives continuous warning information within 7 days before the occurrence of landslide. If emergency preparation time is set to 7 days, warning intensity is $100 \%$ and error warning rate is 0 of the warning model which indicate the model has good timeliness and anti-interference. At the same time, prediction consistency rate is $61 \%$; the model can gradually accumulate confidence in the forecast process while satisfying the above two criteria.

3.3. Optimization of Warning Model. The average value and standard deviation are unchanged for a specific data sample, so the boundary point is only determined by standard deviation multiple. The larger the standard deviation multiple, the stricter the requirements for judging as dangerous. The model responds slowly to abnormal data, and timeliness of the warning model is poor correspondingly. On the contrary, the smaller the standard deviation multiple, the looser the requirements for judging as dangerous. The model responds sensitively to abnormal data, and error warning rate of the warning model is high correspondingly. For a specific standard deviation multiple, the generalization of transition matrix will be insufficient if the sample size is too small. Correspondingly, it cannot fully reflect recent state transition law and will lead to increased prediction sensitivity and error warning rate. On the contrary, abnormal data will impact weakly on prediction if the sample size is too large. But it reduces the sensitivity of the 


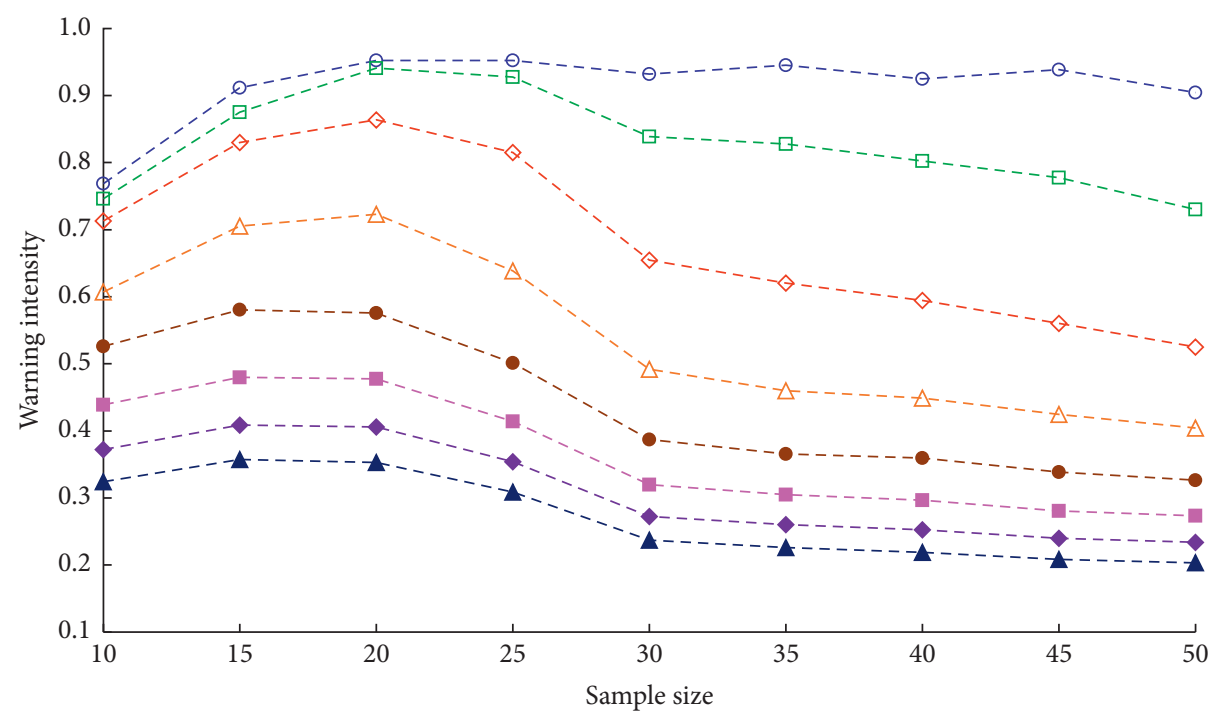

$-\Theta-$ Warning intensity of 1 day

$-\diamond-$ Warning intensity of 5 days

$-\boxminus-$ Warning intensity of 3 days

- - Warning intensity of 9 days

- - Warning intensity of 13 days

-A- Warning intensity of 7 days

- Warning intensity of 11 days

-A- Warning intensity of 15 days

FIGURE 2: Warning intensity with different sample sizes.

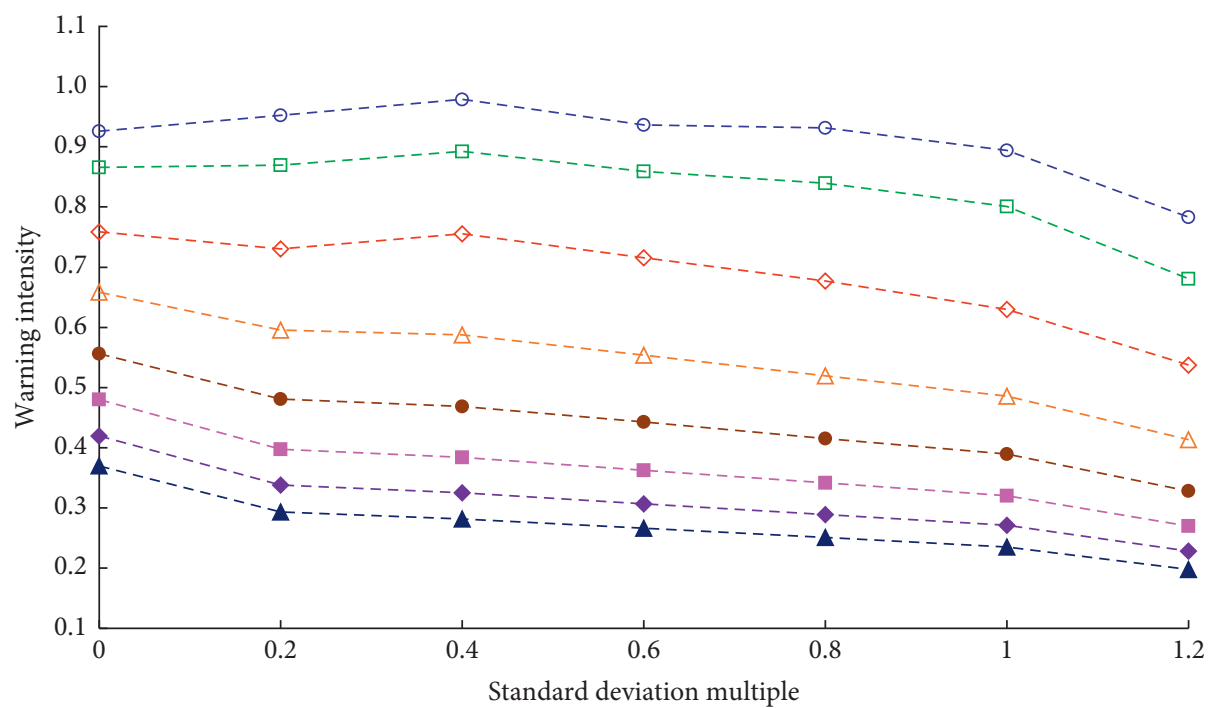

$-\ominus-$ Warning intensity of 1 day

$-\diamond-$ Warning intensity of 5 days

$-\boxminus-$ Warning intensity of 3 days

$-{ }^{-}$- Warning intensity of 7 days

- - Warning intensity of 9 days

- - Warning intensity of 13 days

- - Warning intensity of 11 days

- $\mathbf{-}$ - Warning intensity of 15 days

FIgURE 3: Warning intensity with different standard deviation multiples.

model and will lead to expressing poor timeliness of the warning model. In order to determine optimization parameters of the warning model, use prediction method to analyze each monitoring point in landslide area and optimize sample size and standard deviation multiple from three aspects of timeliness, anti-interference, and reliability. The curves of warning intensity under different sample sizes and standard deviation multiples are shown in Figures 2 and 3; the curves of prediction consistency are shown in Figure 4.
It can be seen from Figure 2 that warning intensity shows a trend of early increasing and then decreasing with increasing of sample size when the standard deviation multiple is unchanged. The optimization sample size is selected to be 20 according to the law of curve fitting. It can be seen from Figure 3 that warning intensity of 1 day, 3 days, and 5 days increases only with increasing of standard deviation multiple from 0.2 to 0.4 , and the others all decrease in the condition of increasing standard deviation multiple. Therefore, alternative options of standard deviation multiple are $0,0.2$, and 0.4 . 


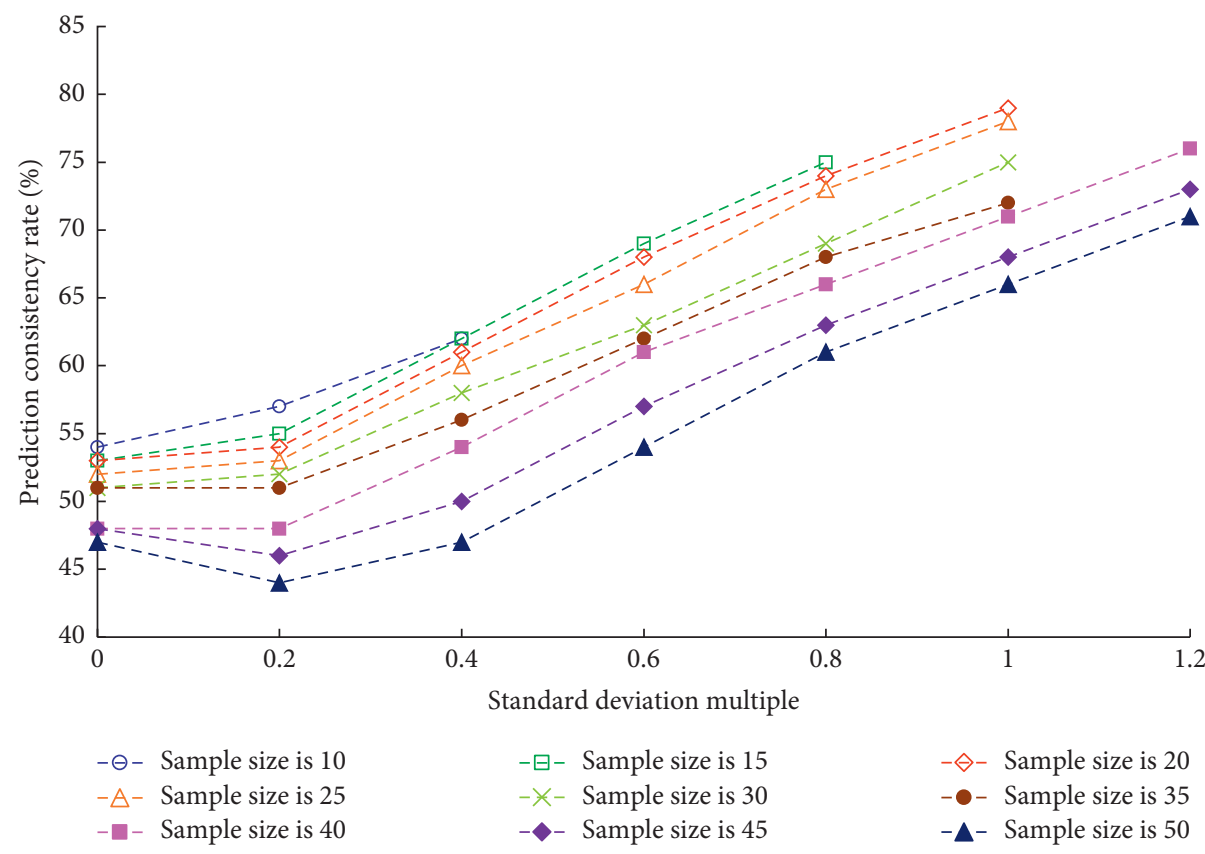

Figure 4: Prediction consistency rate with different standard deviation multiples.

TABLE 2: Warning results of monitoring points within 10 days before landslide.

\begin{tabular}{|c|c|c|c|c|c|c|c|c|c|c|c|}
\hline \multirow{2}{*}{ Serial number of monitoring points } & \multicolumn{10}{|c|}{ Warning days before landslide } & \multirow{2}{*}{ The location of monitoring points } \\
\hline & 10 & 9 & 8 & 7 & 6 & 5 & 4 & 3 & 2 & 1 & \\
\hline $2200-438$ & - & - & - & - & 6 & 5 & 4 & - & 2 & 1 & \multirow{30}{*}{ In the landslide area } \\
\hline $2250-438$ & - & - & - & - & - & 5 & 4 & 3 & 2 & 1 & \\
\hline $2300-438$ & - & - & - & - & - & 5 & 4 & 3 & 2 & 1 & \\
\hline $2350-444$ & - & - & - & 7 & 6 & 5 & 4 & 3 & 2 & 1 & \\
\hline $0-442$ & - & - & - & - & 6 & 5 & 4 & 3 & 2 & 1 & \\
\hline $3200-441$ & - & - & - & - & 6 & 5 & 4 & 3 & 2 & 1 & \\
\hline $3220-441$ & - & - & - & 7 & 6 & 5 & 4 & 3 & 2 & 1 & \\
\hline $2150-462$ & - & - & - & & 6 & 5 & - & 3 & 2 & 1 & \\
\hline $2250-462$ & - & - & - & - & - & - & 4 & 3 & 2 & 1 & \\
\hline $2300-462$ & - & - & - & - & - & - & 4 & 3 & 2 & 1 & \\
\hline $0-461$ & - & - & - & 7 & 6 & - & 4 & 3 & 2 & 1 & \\
\hline $3200-461$ & - & - & - & 7 & 6 & 5 & 4 & 3 & 2 & 1 & \\
\hline $3250-461$ & - & - & - & 7 & 6 & 5 & 4 & 3 & 2 & 1 & \\
\hline $2250-512$ & - & - & - & 7 & 6 & 5 & 4 & 3 & 2 & 1 & \\
\hline $2300-512$ & - & - & - & - & - & - & 4 & 3 & 2 & 1 & \\
\hline $0-512$ & - & - & - & - & - & 5 & 4 & 3 & 2 & 1 & \\
\hline $3300-528$ & - & - & - & 7 & - & 5 & 4 & 3 & 2 & 1 & \\
\hline $3300-515$ & - & - & - & 7 & 6 & 5 & 4 & 3 & 2 & 1 & \\
\hline $2300-548$ & - & - & - & - & - & - & 4 & 3 & 2 & 1 & \\
\hline $0-548$ & - & - & - & - & - & - & 4 & 3 & 2 & 1 & \\
\hline $3300-548$ & - & - & - & - & - & 5 & 4 & 3 & 2 & 1 & \\
\hline Warning rate & 0 & 0 & 0 & 0.38 & 0.52 & 0.71 & 0.95 & 0.95 & 1 & 1 & \\
\hline $2100-438$ & - & - & - & - & - & - & - & - & - & - & \\
\hline $2150-438$ & - & - & - & - & - & - & - & - & - & - & \\
\hline $2100-462$ & - & - & - & - & - & - & - & - & - & - & \\
\hline $3550-470$ & - & - & - & - & - & - & - & - & - & - & \\
\hline $2200-512$ & - & - & - & - & - & - & - & - & - & 1 & \\
\hline $3500-529$ & - & - & - & - & - & - & - & - & - & - & \\
\hline $2250-548$ & - & - & - & - & - & - & - & - & - & 1 & \\
\hline Warning rate & 0 & 0 & 0 & 0 & 0 & 0 & 0 & 0 & 0 & 0.29 & \\
\hline
\end{tabular}



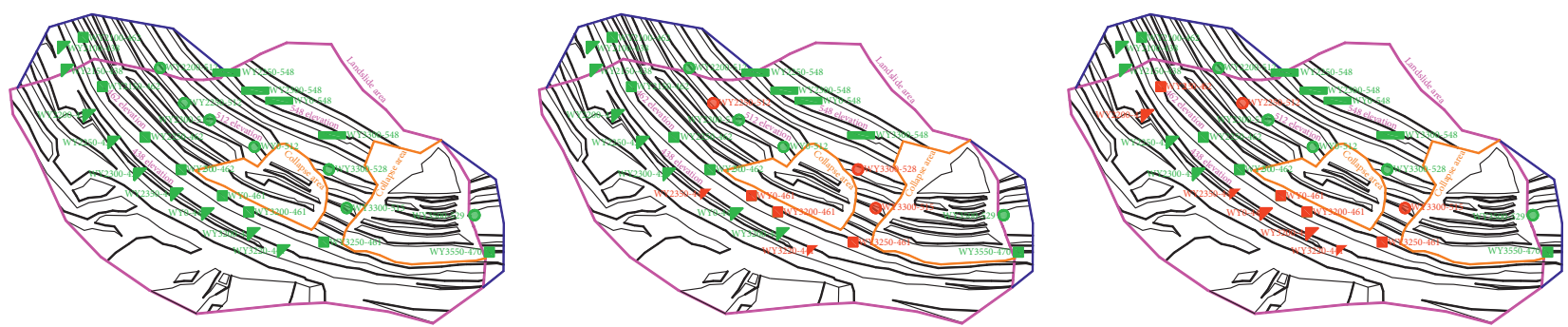

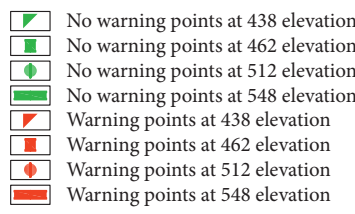

(a)
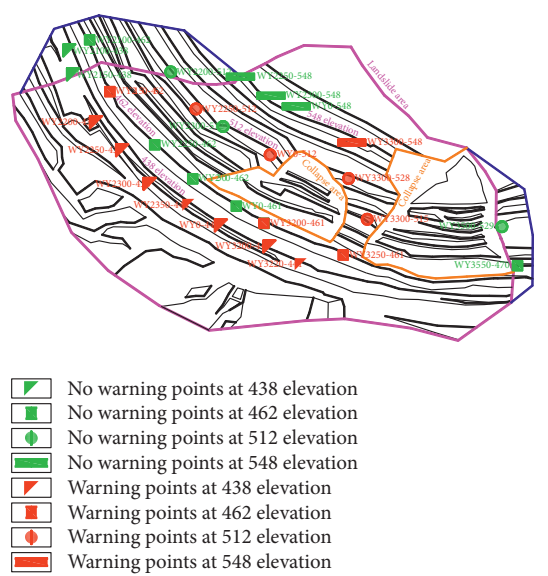

(d)
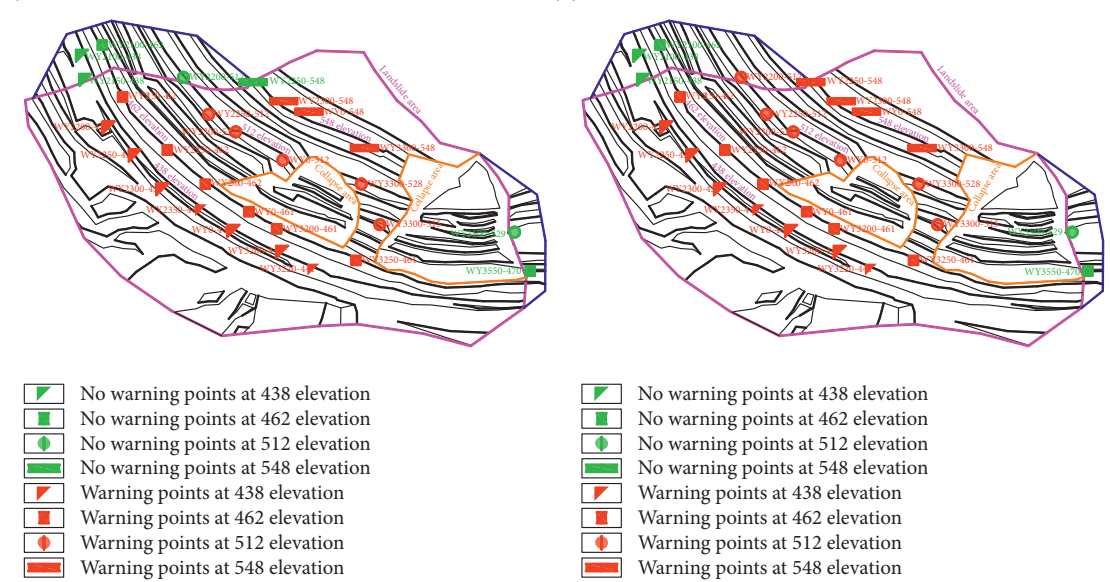

(g)

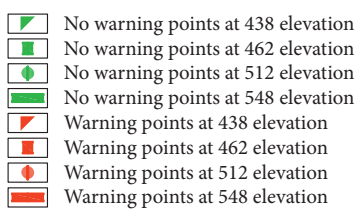

(b)
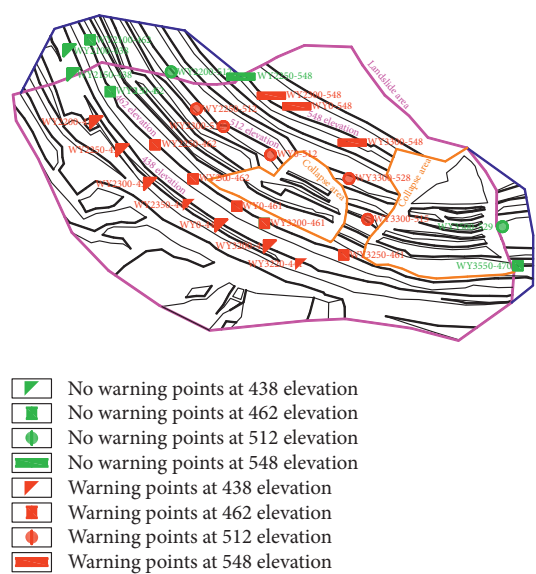

(e)

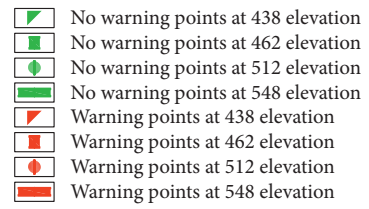

(c)
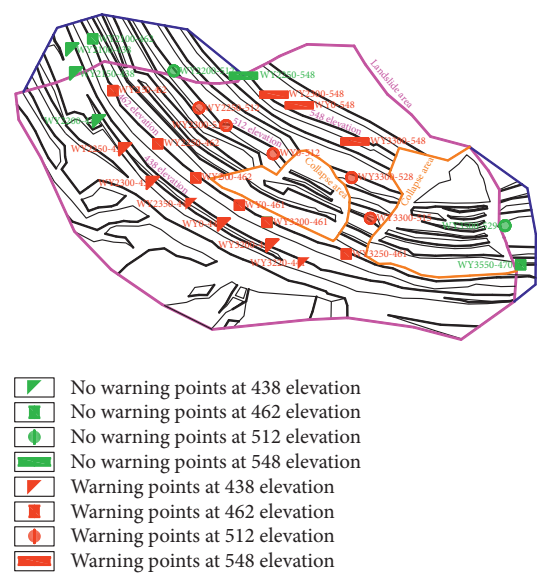

(f) 
It can be seen from Figure 4 that prediction consistency rate gradually increases with increasing of standard deviation multiple when standard deviation multiple is greater than 0.2. This is because increasing of standard deviation multiple leads to respond slowly to abnormal data of the warning model, so the warning model does not need to pursue too high prediction consistency rate. The optimization parameters of the warning model are determined to be sample size of 20 and standard deviation multiple of 0.4 .

3.4. Analysis of Disaster Space-Time Evolution. Warning effect of the model for all monitoring points in the condition of sample size of 20 and standard deviation multiple of 0.4 is shown in Table 2.

The gray in Table 2 indicates that the model gives warning information. It can be seen that warning rate on 4 days and 3 days before occurrence of landslide is as high as $95 \%$ and warning rate on 2 days and 1 day before occurrence of landslide reaches $100 \%$ in the landslide area, which indicates the warning model has good timeliness. On the contrary, warning rate on 1 day before occurrence of landslide is only $29 \%$ and warning rate is 0 for the rest of the time outside the landslide area, which indicates the warning model has good anti-interference. The warning situations of all monitoring points in the slope area before occurrence of landslide are shown in Figure 5.

Figure 5 reveals the space-time evolution of landslide disaster. It can be seen from Figure 5 that the landslide disaster starts at 438 level, upper sliding body loses its support and landslide occurs due to lower sliding, and it belongs to a typical traction landslide.

\section{Conclusion}

This paper established a landslide warning model based on Markov chain theory and optimized parameters, evaluated accuracy of the landslide warning model through a typical landslide disaster case, and revealed the space-time evolution of landslide disaster. The main conclusions are as follows:

(1) This paper analyzed Markov chain theory and proposed a prediction method by assigning time characteristics to the sample and then established a landslide warning model based on Markov chain theory and slope engineering practice.

(2) In order to evaluate accuracy of the landslide warning model, this paper proposed warning model evaluation indicators from the aspects of timeliness, anti-interference, and credibility combined with practical requirements of slope engineering.

(3) The landslide warning model was applied to practice of typical landslide. The model parameters are optimized in combination with evaluation indicators. The optimization parameters of the warning model were determined to be of a sample size of 20 and standard deviation multiple of 0.4 .
(4) In the condition that the warning model obtained optimization parameters, warning results before landslide of each monitoring point have been described, and space-time evolution of landslide disaster has been revealed based on warning situation of each monitoring point.

\section{Data Availability}

The data used to support the findings of this study are available from the corresponding author upon request.

\section{Conflicts of Interest}

The author declares no conflicts of interest.

\section{Acknowledgments}

This paper was supported by research funding of Huanghe Science and Technology College (02032293) and Scientific and Technological Project in Henan Province (202102310567).

\section{References}

[1] N. Bar, M. Kostadinovski, M. Tucker et al., "Rapid and robust slope failure appraisal using aerial photogrammetry and 3D slope stability models," International Journal of Mining Science and Technology, vol. 30, no. 5, pp. 651-658, 2020.

[2] H. Zhao, Y. Tian, Q. Guo, M. Li, and J. Wu, "The slope creep law for a soft rock in an open-pit mine in the Gobi region of Xinjiang, China," International Journal of Coal Science \& Technology, vol. 7, no. 2, pp. 371-379, 2020.

[3] Q.-X. Meng, W.-Y. Xu, H.-L. Wang, X.-Y. Zhuang, W.-C. Xie, and T. Rabczuk, "DigiSim - an open source software package for heterogeneous material modeling based on digital image processing," Advances in Engineering Software, vol. 148, Article ID 102836, 2020.

[4] Z. Tao, C. Zhu, M. He, and M. Karakus, "A physical modelingbased study on the control mechanisms of Negative Poisson's ratio anchor cable on the stratified toppling deformation of anti-inclined slopes," International Journal of Rock Mechanics and Mining Sciences, vol. 138, Article ID 104632, 2021.

[5] Y. Wang, W. K. Feng, R. L. Hu, and C. H. Li, "Fracture evolution and energy characteristics during marble failure under triaxial fatigue cyclic and confining pressure unloading (FC-CPU) conditions," Rock Mechanics and Rock Engineering, vol. 54, no. 2, pp. 799-818, 2021.

[6] M. Saito, "Forecasting the time of occurrence of a slope failure," Proceedings of the 6th International Conference on Soil Mechanics and Foundation Engineering, vol. 6, no. 24, pp. 537-541, 1965.

[7] Z. Wang, X. Yang, and A. Li, "Upper bound limit stability analysis for soil slope with nonuniform multiparameter distribution based on discrete algorithm," Advances in Civil Engineering, vol. 2020, no. 4, 9 pages, 2020.

[8] B. Li, R. Bao, Y. Wang et al., "Permeability evolution of twodimensional fracture networks during shear under constant normal stiffness boundary conditions," Rock Mechanics and Rock Engineering, vol. 54, no. 3, pp. 1-20, 2021.

[9] W.-D. Wu, J.-B. Bai, X.-Y. Wang, S. Yan, and S.-X. Wu, "Numerical study of failure mechanisms and control techniques for a gob-side yield pillar in the sijiazhuang coal mine, 
China," Rock Mechanics and Rock Engineering, vol. 52, no. 4, pp. 1231-1245, 2019.

[10] C. Zhu, M. He, M. Karakus, X. Zhang, and Z. Tao, "Numerical simulations of the failure process of anaclinal slope physical model and control mechanism of negative Poisson's ratio cable," Bulletin of Engineering Geology and the Environment, vol. 80, no. 4, pp. 3365-3380, 2021.

[11] W. Pan, W. Pan, J. Luo et al., "Slope stability of increasing height and expanding capacity of south dumping site of Hesgoula coal mine: a case study," International Journal of Mining Science \& Technology, vol. 30, 2020.

[12] R. Supper, D. Ottowitz, B. Jochum et al., "Geoelectrical monitoring: an innovative method to supplement landslide surveillance," Near Surface Geophysics, vol. 12, no. 1, pp. 133-150, 2015.

[13] D. Guo and M. Hamada, "Qualitative and quantitative analysis on landslide influential factors during Wenchuan earthquake: a case study in Wenchuan County," Engineering Geology, vol. 152, no. 1, pp. 202-209, 2013.

[14] H. B. Wang, S. R. Wu, J. S. Shi, and B. Li, "Qualitative hazard and risk assessment of landslides: a practical framework for a case study in China," Natural Hazards, vol. 69, no. 3, pp. 1281-1294, 2013.

[15] A. Smith and N. Dixon, "Quantification of landslide velocity from active waveguide-generated acoustic emission," Canadian Geotechnical Journal, vol. 52, no. 4, pp. 413-425, 2015.

[16] F. Ciervo, G. Rianna, P. Mercogliano, and M. N. Papa, "Effects of climate change on shallow landslides in a small coastal catchment in southern Italy," Landslides, vol. 14, no. 3, pp. 1043-1055, 2016.

[17] F. Yan, Q. Zhang, S. Ye, and B. Ren, “A novel hybrid approach for landslide susceptibility mapping integrating analytical hierarchy process and normalized frequency ratio methods with the cloud model," Geomorphology, vol. 327, no. 2, pp. 170-187, 2019.

[18] I. Emanuele, B. Federica, F. Riccardo et al., "Big data managing in a landslide early warning system: experience from a ground-based interferometric radar application," Natural Hazards \& Earth System Sciences, vol. 17, no. 10, pp. 1-22, 2017.

[19] D. Tiranti and D. Rabuffetti, "Estimation of rainfall thresholds triggering shallow landslides for an operational warning system implementation," Landslides, vol. 7, no. 4, pp. 471-481, 2010.

[20] E. Canli, B. Loigge, and T. Glade, "Spatially distributed rainfall information and its potential for regional landslide early warning systems," Natural Hazards, vol. 91, no. S1, pp. 103-127, 2018.

[21] C. Lian, Z. Zeng, W. Yao, and H. Tang, "Ensemble of extreme learning machine for landslide displacement prediction based on time series analysis," Neural Computing and Applications, vol. 24, no. 1, pp. 99-107, 2014.

[22] V. Tofani, C. Del Ventisette, S. Moretti, and N. Casagli, "Integration of remote sensing techniques for intensity zonation within a landslide area: a case study in the northern apennines, Italy," Remote Sensing, vol. 6, no. 2, pp. 907-924, 2014.

[23] R. Supper, D. Ottowitz, B. Jochum et al., "Geoelectrical monitoring: an innovative method to supplement landslide surveillance and early warning," Near Surface Geophysics, vol. 12, no. 1, pp. 133-150, 2014.

[24] D. G. Sutherland, M. Hansler Ball, S. J. Hilton, and T. E. Lisle, "Evolution of a landslide-induced sediment wave in the navarro river, California," Geological Society of America Bulletin, vol. 114, no. 8, pp. 1036-1048, 2002.

[25] F. Dammeier, J. R. Moore, C. Hammer, F. Haslinger, and S. Loew, "Automatic detection of alpine rockslides in continuous seismic data using hidden Markov models," Journal of Geophysical Research Earth Surface, vol. 121, no. 2, pp. 351371, 2016.

[26] M. Peng, X. Y. Li, D. Q. Li, S. H. Jiang, and L. M. Zhang, "Slope safety evaluation by integrating multi-source monitoring information," Structural Safety, vol. 49, pp. 65-74, 2014.

[27] G. J. Dick, E. Eberhardt, A. G. Cabrejolievano et al., "Development of an early-warning time-of-failure analysis methodology for open-pit mine slopes utilizing ground-based slope stability radar monitoring data," Canadian Geotechnical Journal, vol. 52, no. 4, pp. 1-15, 2014. 